\title{
Corrección de genu valgo postraumático con osteotomía de cierre en tibia proximal
}

\section{High Tibial Osteotomy for Posttraumatic Genu Valgus Deformity Correction}

\author{
José Ignacio Laso ${ }^{1} \quad$ Carlos Alejandro Rojas ${ }^{2}$ Álvaro Fernando Valenzuela ${ }^{3}$ Santiago Álvarez ${ }^{4}$ \\ Nicolás Santiago Gaggero 3
}

${ }^{1}$ Department of Orthopedic, Universidad de Chile, Hospital del Trabajador, Santiago de Chile, Chile

${ }^{2}$ Department of Orthopedic, Universidad Andrés Bello, Hospital del

Trabajador, Santiago de Chile, Chile

${ }^{3}$ Knee Unit, Hospital del Trabajador, Santiago de Chile, Chile

4 Universidad de Los Andes, Las Condes, Chile
Address for correspondence Carlos Alejandro Rojas, MD, Department of Orthopedic, Universidad Andrés Bello, Hospital del Trabajador, Santiago de Chile, Chile (e-mail: carlosrojasz@gmail.com).

\section{Resumen}

\section{Palabras Claves}

- complicación

- genu valgo

- osteotomía

- fractura de platillos tibiales
Objetivo Presentar un caso de complicación de fractura de platillos tibiales Schatzker VI y su manejo quirúrgico.

Material y Método Se presenta un caso de fractura de platillos tibial Schatzker VI que evoluciona con malalineamiento en valgo secundario a hundimiento del platillo tibial, junto a una revisión de la literatura y la descripción del manejo quirúrgico.

Resultados Se realizó una osteotomía en cuña de cierre medial de tibia proximal y se estabilizó con placa bloqueada (TomoFix), con una corrección completa de la deformidad sin complicaciones.

Discusión La osteotomía en cuña de cierre medial en tibia proximal es una técnica descrita en el manejo de artrosis secundaria a malalineamiento en valgo de la rodilla. Mediante dos osteotomías iniciadas por la cortical medial hacia la lateral con un fulcro esa última, se retira una cuña de dimensiones conocidas y se mantiene la reducción con algún elemento de osteosíntesis. De esa forma, se permite la corrección angular de la deformidad, previamente planificada. Los resultados en distintas series son en general favorables.

Conclusión El malalineamiento de la extremidad posterior a una fractura de platillos tibiales y la consecuente sobrecarga del compartimento afectado en la rodilla, asociado al daño articular, evoluciona con degeneración articular que termina en una artrosis unicompartimental secundaria, la cual puede ser prevenida con el uso de osteotomías correctoras de ejes como la osteotomía de tibia proximal, permitiendo normalizar la distribución de las cargas en los compartimentos mediante la corrección del eje mecánico alterado y así prolongar la sobrevida articular.

Nivel de Evidencia Tipo IV, Caso Clínico. received

January 30, 2017

accepted

October 2, 2017

published online

October 25, 2017
DOI https://doi.org/

$10.1055 / \mathrm{s}-0037-1607461$ ISSN $0716-4548$.
Copyright $\odot 2017$ by Thieme Revinter

License terms

Publicações Ltda, Rio de Janeiro, Brazil
ㄷ) (i) $\odot$ (\$) 
Abstract

\section{Keywords \\ - complication \\ - genu valgus \\ - osteotomy \\ - tibial plateau fracture}

Objective To present a Schatzker VI tibial plateau fracture case complication and its surgical management

Methods We present a case of tibial plateau fracture, type VI according to Schatzkeŕs classification, that developed limb malalignment secondary to tibial plateau depression resulting in a genu valgum deformity, along with a literature review and a brief description of the surgical technique.

Results High tibial medial closing wedge osteotomy, stabilized with locking plate (tomoFix) was performed, with a complete correction of the deformity without complications.

Discussion High tibial medial closing wedge is a known procedure used in the management of valgus knee malalignment secondary osteoarthritis. By means of two osteotomies made from medial to lateral cortices, using the latter as a fulcrum, a wedge with known dimensions is subtracted and reduction is maintained with some osteosynthesis element. In this fashion, it allows the previously planned angular correction. Results in different reports are mostly favorable.

Conclusion The malalignment of the limb after a tibial plateau fracture and the consequent overload of the affected knee compartment; associated with joint damage, evolves in joint degeneration and eventually, in a secondary unicompartmental osteoarthritis. This can be avoided with the use of corrective osteotomies such as the proximal tibial osteotomy, which allows a proper distribution of loads in the compartments by correcting the altered mechanical axis and thus, prolonging joint survival.

Level of Evidence Type IV, Clinical Case.

\section{Introducción}

Las fracturas de platillos tibiales es una lesión ósea de la epífisis proximal de la tibia. Se producen por mecanismos de alta energía en pacientes jóvenes, y en general, se asocia a lesiones intra y extrarticulares. La clasificación que ampliamente se utiliza para clasificar ese tipo de lesión, es la descrita por Schatzker y col. ${ }^{1}$

La artrosis temprana es una complicación frecuente en esos pacientes. Ella puede estar dada por el daño articular al momento de la lesión, una alteración en la alineación de ejes, e incluso por una lesión de las estructuras estabilizadoras de la articulación. ${ }^{2}$

Para el tratamiento de la artrosis postraumática, se describen diferentes técnicas quirúrgicas que permiten corregir la alteración causante de esa degeneración articular precoz y de la artrosis en sí misma.

En el caso de artrosis secundaria a una alteración de ejes en la extremidad, una alternativa de manejo es el uso de osteotomías correctoras de ejes, siendo ampliamente recomendadas las osteotomías a nivel del fémur distal y con menor frecuencia, las de tibia proximal.

El objetivo de este trabajo es presentar un caso de complicación de fractura de platillos tibiales Schatzker VI, debido a un mal alineamiento post reducción, manejado mediante una osteotomía de cierre medial de tibia proximal.

\section{Caso Clínico}

Se presenta el caso de un paciente masculino, de 51 años de edad, sin antecedentes médicos de relevancia, el cual sufre fractura de platillos tibiales Schatzker tipo VI en rodilla derecha, asociado a fractura de pierna ipsilateral y fractura de platillos tibiales Schatzker I contralateral. El paciente fue tratado inicialmente en otro centro hospitalario. En la rodilla derecha se realizó una reducción y osteosíntesis con doble placa de ambos platillos, las fracturas de fíbula y de tibia diafisiaria fueron resueltas con placas de osteosíntesis, las cuales fueron retiradas al año de evolución, la extremidad contralateral fue resuelta mediante tornillos canulados. El paciente evoluciona con una artrosis del compartimento femorotibial lateral asociado a un mal alineamiento en valgo de la extremidad inferior derecha.

Evaluado en nuestro centro a los 2 años de evolución. En el examen físico destaca un genu valgo en pierna derecha, una rodilla con rango de movilidad completo, bostezo lateral $(++/+++)$, sin signos de inestabilidad anteroposterior.

En el estudio imagenológico destaca: artrosis del compartimento femorotibial lateral grado 2 de Kellgren and Lawrence, asociado a una fractura consolidada de platillos tibiales con material de osteosíntesis in situ (-Fig. 1), en la telerradiografía, destaca un eje mecánico en valgo de $11^{\circ}$ con un ángulo tibial proximal medial de $95^{\circ}$ (-Fig. 2).

Se realiza una cirugía en dos tiempos quirúrgicos. En el primero, se realiza la retirada de los materiales de osteosíntesis y una artroscopía diagnóstica, la cual descarta cambios degenerativos a nivel del compartimiento femorotibial medial. Fue dado de alta al segundo día postoperatorio, sin complicaciones. El segundo tiempo quirúrgico fue realizado a los 3 meses postoperatorios. La cirugía definitiva consistió en la realización de osteotomía en cuña de cierre medial de tibia de $10 \mathrm{~mm}$, la cual fue estabilizada con placa TomoFix (DePuy Synthes) de tibia proximal (-Fig. 3). El paciente evoluciona 


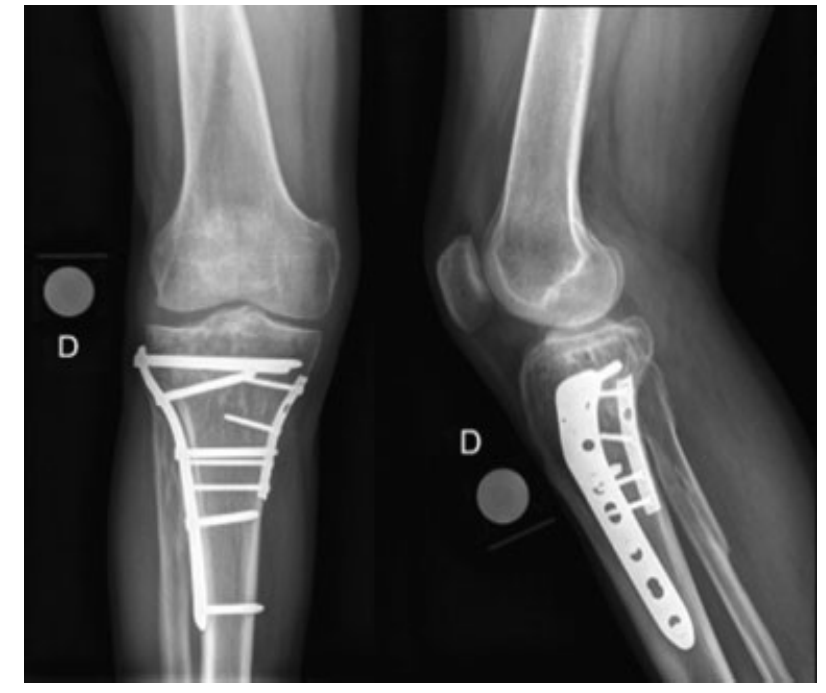

Fig. 1 Radiografía anteroposterior (AP) y lateral (L) de rodilla derecha al ingreso. Se aprecia osteosíntesis previa con doble placa para fractura de platillos tibiales.

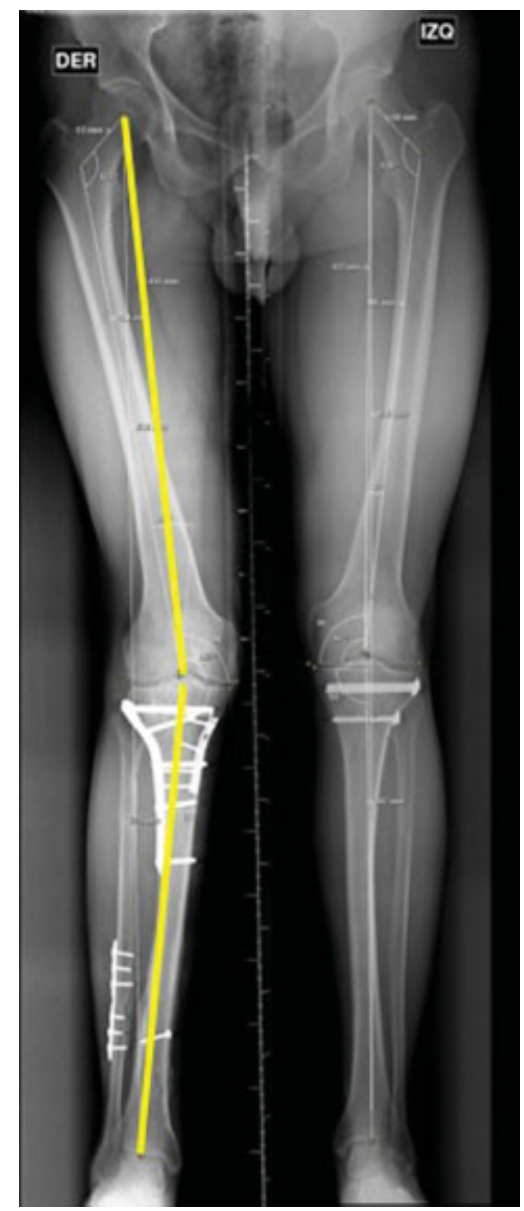

Fig. 2 Telerradiografía AP de extremidades inferiores, eje mecánico con $11^{\circ}$ de valgo a derecha y de $1^{\circ}$ a izquierda.

favorablemente, dado de alta al segundo día postoperatorio con carga mínima, aumentando la carga progresivamente hasta lograr carga completa a las 12 semanas postoperatorias. Al control de imágenes en las 8 semanas post operatorias

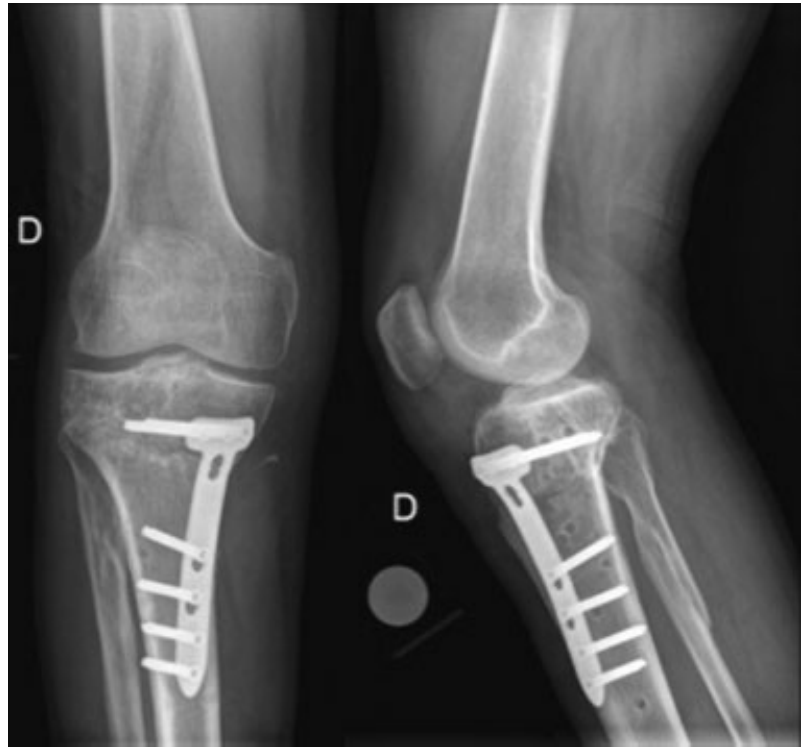

Fig. 3 Radiografías de rodilla derecha AP y L postoperatorias, se aprecia osteotomía de cierre medial tibial y osteosíntesis con placa bloqueada.

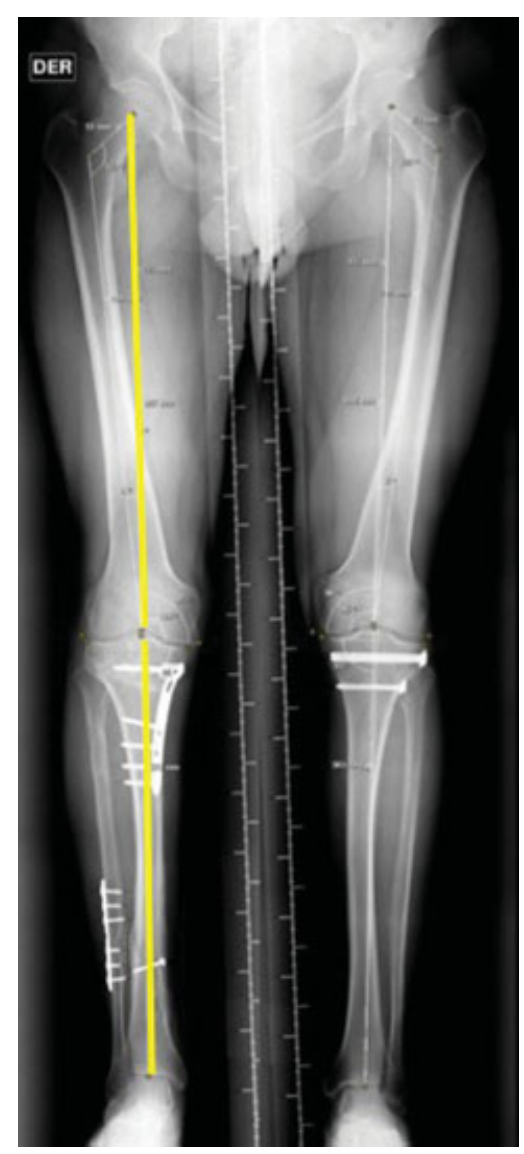

Fig. 4 Telerradiografía de extremidades inferiores postoperatoria con eje mecánico derecho corregido a neutro $\left(0^{\circ}\right)$.

mediante telerradiografía, se aprecia ángulo de eje mecánico corregido de $0^{\circ}$ y un ángulo tibial proximal medial de $85^{\circ}$ (-Fig. 4). El paciente evoluciona asintomático con rango de movilidad completo al año de seguimiento. 


\section{Discusión}

Las fracturas de platillos tibiales corresponden al $1 \%$ de las fracturas, y al 8\% de las fracturas en adultos mayores. Su distribución etaria es bimodal, en jóvenes son producto de traumatismos de alta energía como accidentes de tránsito o caídas de altura, con mecanismo de varo o valgo y caídas a nivel en adultos hombres mayores de 50 años y en mujeres mayores de 70 años. La ubicación más frecuente es en el platillo lateral, seguido por fractura de ambos platillos y con menor frecuencia la fractura del platillo medial. ${ }^{3}$ Esas fracturas, se asocian a lesiones meniscales y ligamentarias en un $20-50 \%$ de los casos según el tipo de fractura en distintas series. ${ }^{1,4,5}$ La clasificación más usada es la descrita por Schatzker que las agrupa en 6 tipos según los hallazgos imagenológicos en relación al rasgo de fractura. ${ }^{5}$ Existe hasta un $10 \%$ de esas lesiones que no son clasificables según el sistema propuesto por Schatzker, en las que se puede utilizar la clasificación de Hohl y Moore. ${ }^{6}$ Otra clasificación complementaria a las anteriores es la descrita por Luo, la cual permite mediante el uso de tomografía computarizada, clasificar esas fracturas dividiendo la superficie articular del corte axial en tres columnas y orientar la reducción de los fragmentos mediante ese sistema. $^{7}$

Una de las complicaciones agudas más severas es el síndrome compartimental el cual siempre se debe sospechar y descartar en ese tipo de pacientes, ${ }^{3}$ sobre todo en aquellos con fracturas tipo Schatzker VI en que alcanza hasta un $18 \% .^{8}$ En cuanto a las complicaciones crónicas, la más frecuente es las artrosis, presente hasta en un 31\% seguida del mal alineamiento de ejes en varo o valgo lo que puede contribuir secundariamente al desarrollo precoz de artrosis. ${ }^{9}$ El colapso del platillo lateral luego de una reducción adecuada y posterior desarrollo de malalineamiento en valgo se debe, en parte, a la presencia de hueso subcondral de menor densidad que en el del platillo medial. Esa diferencia en la densidad se explica por la carga proporcional que resiste cada platillo. ${ }^{10}$ Así, el hueso dañado tendría una menor resistencia a la carga y asociado al defecto propio de la fractura se produciría ese colapso.

Cuando una fractura consolida con malalineamiento, ya sea por cambios en la longitud, alineamiento, rotación o traslación de los segmentos, se altera la distribución de cargas en la rodilla y se producirá un desgaste acelerado del cartílago del compartimento afectado, que en el caso del compartimento femorotibial lateral, puede evolucionar con genu valgo como ocurrió en ese caso.

El valgo postraumático, es poco frecuente y se observa principalmente en fracturas de platillo tibial lateral y en fracturas de ambos platillos. Clínicamente, se manifiesta con dolor en el compartimiento lateral de la rodilla, y puede observarse un aumento en la distancia intermaleolar mayor a $5 \mathrm{~cm}$. Radiológicamente hay un aumento del ángulo de eje mecánico por sobre los 5 grados. $^{11}$

Dentro de las opciones de manejo, existe el conservador para angulaciones menores, osteotomías (femoral y tibial) y artroplastia total o parcial de rodilla, reservada generalmente para pacientes de mayor edad y con asociación a artrosis sintomática. ${ }^{12,13}$
En el tratamiento conservador se describe el uso de fármacos como AINEs y acetaminofeno, ese último validado por la AAOS con recomendación moderada de su uso en artrosis de rodilla en 2008 y posteriormente con recomendación no conclusiva en 2013. ${ }^{14}$ El uso de órtesis también aparece como una alternativa, sin embargo, la evidencia que apoye esa medida, indica que los beneficios serían limitados en el manejo sintomático y nulos en el control de la progresión de la enfermedad. ${ }^{15,16}$

En el manejo quirúrgico hay cierto consenso para la indicación de una osteotomía correctora de eje; paciente menor de 50 años, activo, sin artrosis en compartimento patelofemoral, con índice de masa corporal menor a 30, con extensión completa de rodilla y flexión sobre $90^{\circ}$, sin contractura en flexión o inferior a $10^{\circ} .^{17}$

Para determinar en qué segmento de la extremidad está ubicada la deformidad, deben medirse los ángulos tibial proximal medial (ATPM) y femoral distal lateral (AFDL), cuyos valores normales oscilan entre $85^{\circ}$ y $90^{\circ}$ (media $87.5^{\circ}$ ). Si el ATPM mide más de $90^{\circ}$, la deformidad corresponde a un valgo secundario a la tibia, si él mide menos de $85^{\circ}$, la deformidad será un varo. En deformidades secundarias a alteraciones femorales, estaremos frente a un valgo si el AFDL mide menos de $85^{\circ}$ y se tratará de un varo si ese ángulo mide más de $90^{\circ}$. Una vez localizada la deformidad, cabe planificar la localización de la osteotomía a realizar. Ese punto es de suma importancia y el cirujano debe planificar con esa información, la cirugía que se realizará. La osteotomía tibial está reservada para deformidades localizadas en la tibia. A su vez, las osteotomías femorales se proponen para el manejo de deformidades femorales. De no seguir esa regla, es probable que al corregir el malalineamiento se produzca una oblicuidad de la superficie articular secundaria. ${ }^{18}$

La osteotomía varizante proximal de tibia está indicada principalmente para casos de genu valgo leve, que no sobrepasen los $12^{\circ}$ de angulación y para aquellos en que la deformidad en valgo está situada en el extremo proximal de la tibia, con cóndilos femorales alineados correctamente, como es el caso de la mayoría de las fracturas de platillos tibiales con componente de hundimiento. ${ }^{13,17,18}$

Para la planificación quirúrgica se utilizan telerradiografías de extremidades inferiores donde se debe determinar el ángulo de la deformidad y calcular el ángulo a corregir con la osteotomía. Ese último se obtiene con el método descrito por Dugdale: se marca la línea de carga en un punto ubicado al 50-54\% de la superficie de los platillos tibiales, de medial a lateral, si no existe patología degenerativa del compartimento femorotibial medial, en caso contrario se ubica en el 62\% (de esa forma descargando al compartimento medial). Luego ese punto se une con una línea al centro de la cabeza femoral y con otra al centro del talo. El ángulo formado entre esas dos líneas corresponde al ángulo a corregir. Eso último es válido tanto para la osteotomía femoral como para la osteotomía tibial proximal. ${ }^{19,20}$

La técnica de osteotomía de cierre medial tibial, implica realizar la osteotomía en la cara medial de la tibia, a través de un abordaje anteromedial, $1 \mathrm{~cm}$ proximal al tubérculo de la tibia. Se secciona la porción superficial del ligamento colateral medial. Posteriormente se prepara y marca la zona de la osteotomía proximal, ubicada levemente proximal a la tuberosidad de la 
tibia. Para ello se utilizan dos agujas de Kirschner como referencia para el corte proximal y dos para el corte distal. Se inicia la osteotomía proximal con orientación horizontal en el plano coronal por la cortical medial y luego las corticales anterior y posterior, dejando al menos $5 \mathrm{~mm}$ intactos hasta la cortical lateral para no comprometerla, ya que actúa como fulcro. Luego se realiza la osteotomía distal de la misma forma, iniciando por la cortical medial, continuando con las corticales anterior y posterior. Luego se reseca la cuña obtenida al unir ambas osteotomías y se estabilizan ambos fragmentos con grapas o placas. El fragmento resecado tiene un poder de corrección angular que es proporcional al tamaño de la base de la cuña, siendo lo más aceptado una relación lineal de un grado por cada milímetro de cuña hasta los $6^{\circ} .^{21}$

La selección de pacientes es clave para un resultado exitoso: pacientes jóvenes, menores de 60 años o con esperanza de vida mayor a 20 años, físicamente activos, con estabilidad ligamentaria y enfermedad unicompartimental, además de rango de flexión previo a la cirugía de al menos $90^{\circ} .^{22}$

Existen diversos resultados en la literatura para el manejo con osteotomía los cuales, en su mayoría, presentan resultados favorables. Marti y col., obtuvo buenos y excelentes resultados en 30 de 36 pacientes con angulación promedio 11,6 grados con seguimiento a 11 años. ${ }^{23}$ Una revisión realizada por Leone y col., de 11 estudios con seguimiento a largo plazo, muestra resultados exitosos entre un $80-95 \%$ de los casos a 5 años y 53-85\% a 10 años plazo, haciendo hincapié en la importancia de la selección de pacientes en que se opta por esa cirugía. ${ }^{13}$ En la serie de Chambat y col., que incluyó 47 pacientes manejados con osteotomía de cierre medial de tibia, un $91 \%$ de los casos tuvo mejoría en cuanto al dolor, con hasta $72 \%$ de resultados buenos o muy buenos. 24

Por último, la artroplastía también aparece como una alternativa de tratamiento. Ella representa varios desafíos al momento de planificar la cirugía y de realizarla, desde el abordaje a utilizar, las dimensiones de las osteotomías, tanto femorales como tibial y el balance de partes blandas. La indicación de reemplazo articular está dada por el grado de degeneración articular, edad del paciente junto a su nivel de actividad y la sintomatología del paciente. Tomando eso en consideración, es importante también saber que los resultados en pacientes sometidos a artroplastia total de rodilla por artrosis post traumática difieren de aquellos con artrosis primaria. En el estudio de Scott y col., la tasa de complicaciones reportada fue superior en los primeros luego de compararla con los reportes previos existentes en la literatura, siendo en orden de prevalencia, complicaciones de herida operatoria hasta un $13 \%$ y rigidez persistente hasta $10 \%{ }^{25}$ Por lo anterior, la indicación de artroplastia total de rodilla debe ser para pacientes seleccionados.

Presentamos un caso de gonartrosis sintomática de compartimiento femorotibial lateral, secundaria malalinemiento en valgo de $11^{\circ}$ por una fractura de platillos tibiales, que fue manejada con osteotomía de cierre medial de tibia proximal con la cual se corrigió la deformidad sin complicaciones a corto ni a mediano plazo. La técnica descrita corresponde a una alternativa en pacientes jóvenes donde el objetivo es la preservación articular, con resultados favorables en un gran porcentaje de casos a cinco y diez años de seguimiento.

\section{Conclusiones}

En las fracturas de platillos tibiales, se debe ser enfático en obtener una adecuada alineación y reducción de la fractura con el objetivo de preservar la superficie articular en el corto y largo plazo. En los casos con importante compromiso del cartílago articular, se debe ser minucioso en evitar complicaciones como en el caso recién descrito en donde el paciente desarrolló una pérdida del eje mecánico, que debe corregirse a tiempo para evitar una sobrecarga del compartimento lesionado y así evitar el proceso degenerativo de la articulación, por lo que la indicación en esos pacientes es la corrección del eje con osteotomía en la tibia, mejorando así la sobrevida de la articulación.

Esa alternativa quirúrgica es una opción válida y con resultados favorables según la literatura revisada.

\section{Conflictos de intereses}

Autores declaran que no hay conflictos de intereses.

\section{Bibliografia}

1 Schatzker J, McBroom R, Bruce D. The tibial plateau fracture. The Toronto experience 1968-1975. Clin Orthop Relat Res 1979;(138): 94-104

2 Furman BD, Olson SA, Guilak F. The development of posttraumatic arthritis after articular fracture. J Orthop Trauma 2006;20(10): 719-725

3 Sassoon A, Summers N, Langford J. Tibial Plateau Fracture. In: Scott WN, editor. Insall \&amp; Scott Surgery of the Knee. II. 6th Edition ed: Elsevier; 2017:1180-1194

4 Abdel-Hamid MZ, Chang CH, Chan YS, et al. Arthroscopic evaluation of soft tissue injuries in tibial plateau fractures: retrospective analysis of 98 cases. Arthroscopy 2006;22(06):669-675

5 Markhardt BK, Gross JM, Monu JU. Schatzker classification of tibial plateau fractures: use of CT and MR imaging improves assessment. Radiographics 2009;29(02):585-597

6 Hohl M, Moore T. Articular Fractures of the Proximal Tibia. In: Evarts C, editor. Surgery of the Musculoskeletal System. 2nd ed. New York: Churchill; 1990

7 Luo CF, Sun H, Zhang B, Zeng BF. Three-column fixation for complex tibial plateau fractures. J Orthop Trauma 2010;24(11):683-692

8 Stark E, Stucken C, Trainer G, Tornetta P III. Compartment syndrome in Schatzker type VI plateau fractures and medial condylar fracture-dislocations treated with temporary external fixation. J Orthop Trauma 2009;23(07):502-506

9 Rademakers MV, Kerkhoffs GM, Sierevelt IN, Raaymakers EL, Marti RK. Operative treatment of 109 tibial plateau fractures: five- to 27year follow-up results. J Orthop Trauma 2007;21(01):5-10

10 Hvid I. Mechanical strength of trabecular bone at the knee. Dan Med Bull 1988;35(04):345-365

11 Backstein D, Morag G, Hanna S, Safir O, Gross A. Long-term followup of distal femoral varus osteotomy of the knee. J Arthroplasty 2007;22(04, Suppl 1):2-6

12 Coventry MB. Proximal tibial varus osteotomy for osteoarthritis of the lateral compartment of the knee. J Bone Joint Surg Am 1987; 69(01):32-38

13 Leone J, Hanssen A. Osteotomy About the Knee. In: Scott W, editor. Insall \&amp; Scott Surgery of the Knee. 5th ed. Philadelphia: Elsevier; 2012:910-925 
14 Jevsevar DS. Treatment of osteoarthritis of the knee: evidence-based guideline, 2nd edition. J Am Acad Orthop Surg 2013;21(09):571-576

15 Duivenvoorden T, Brouwer RW, van Raaij TM, Verhagen AP, Verhaar JA, Bierma-Zeinstra SM. Braces and orthoses for treating osteoarthritis of the knee. Cochrane Database Syst Rev 2015;(03):CD004020

16 van Raaij TM, Reijman M, Brouwer RW, Bierma-Zeinstra SM, Verhaar JA. Medial knee osteoarthritis treated by insoles or braces: a randomized trial. Clin Orthop Relat Res 2010;468(07):1926-1932

17 Stuart M, Backstein D, Logan M, Muerllner T. Osteotomy About the Knee International Roundtable Discussion. In: Scott W, editor. Insall \&amp; Scott Surgery of the Knee. 5th ed. Philadelphia: Elsevier; 2012:944-951

18 Sánchez-Martín M, Ruiz-Valdivieso T. Genu Valgum Artrósico. Tratamiento mediante Osteotomías. Revista Española de Cirugia Osteoarticular. 2008;43(233):20-32

19 Puddu G, Cipolla M, Cerullo G, Franco V, Giannì E. Which osteotomy for a valgus knee? Int Orthop 2010;34(02):239-247
20 Dugdale TW, Noyes FR, Styer D. Preoperative planning for high tibial osteotomy. The effect of lateral tibiofemoral separation and tibiofemoral length. Clin Orthop Relat Res 1992;(274):248-264

21 Noyes F. Tibial and Femoral Osteotomy for Varus and Valgus Knee Syndromes: Diagnosis, Osteotomy Techniques, and Clinical Outcomes. Noyes' Knee Disorders: Surgery, Rehabilitation, Clinical Outcomes. 2nd ed. Philadelphia: Elsevier; 2017:773-847

22 Iorio R, Healy WL. Unicompartmental arthritis of the knee. J Bone Joint Surg Am 2003;85-A(07):1351-1364

23 Marti RK, Verhagen RA, Kerkhoffs GM, Moojen TM. Proximal tibial varus osteotomy. Indications, technique, and five to twenty-oneyear results. J Bone Joint Surg Am 2001;83-A(02):164-170

24 Chambat P, Selmi T, Dejour D, Denoyers J. Varus Tibial Osteotomy. Oper Tech Sports Med 2000;8(01):44-47

25 Windsor R, Walter W. Total Knee Arthroplasty After Fractures About the Knee. In: Bono J, Scott R, editors. Revision Total Knee Arthroplasty. New York: Springer; 2005:237-243 